ANNALES

POLONICI MATHEMATICI

$106(2012)$

\title{
Smoothness of the Green function for a special domain
}

\author{
by Serkan Celik (Gebze) and Alexander Goncharov (Ankara)
}

Dedicated to Professor J. Siciak on the occasion of his 80th birthday

\begin{abstract}
We consider a compact set $K \subset \mathbb{R}$ in the form of the union of a sequence of segments. By means of nearly Chebyshev polynomials for $K$, the modulus of continuity of the Green functions $g_{\mathbb{C} \backslash K}$ is estimated. Markov's constants of the corresponding set are evaluated.
\end{abstract}

1. Introduction. If a compact set $K$ is regular with respect to the Dirichlet problem then the Green function $g_{\mathbb{C} \backslash K}$ of $\mathbb{C} \backslash K$ with pole at infinity is continuous throughout $\mathbb{C}$. We are interested in finding its modulus of continuity. The problem of smoothness of $g_{\mathbb{C} \backslash K}$ near the boundary of $K$ was considered by many authors (see e.g. the references in the survey A2 and more recent [CG], $[\mathrm{RR},[\mathrm{AG}]$ ). A new impulse to investigate smoothness properties of the Green functions came in 2006, after appearance of the monograph $[\mathrm{T}]$ by V. Totik.

Here we consider a special compact set $K \subset \mathbb{R}$ in the form of the union of a sequence of segments. For the corresponding Green function we use the well-known representation

$$
g_{\mathbb{C} \backslash K}(z)=\sup \left\{\frac{\log |P(z)|}{\operatorname{deg} P}: P \in \mathcal{P}, \operatorname{deg} P \geq 1,|P|_{K} \leq 1\right\} .
$$

Here and below, $\mathcal{P}=\bigcup_{n=0}^{\infty} \mathcal{P}_{n}$ where $\mathcal{P}_{n}$ denotes the set of all complex polynomials of degree at most $n,|P|_{K}$ is the supremum norm of $P$ on $K$, and $\log$ denotes the natural logarithm.

There are many sequences of polynomials that realize the supremum above, for example the normalized Fekete polynomials (see e.g. [P. Th. 11.1]), the normalized Chebyshev polynomials with zeros on $K$ (see e.g. Gol, Th. VII.4.4]), or any normalized sequence of polynomials orthogonal with

2010 Mathematics Subject Classification: Primary 31A15; Secondary 41A10, 41A17.

Key words and phrases: Green function, modulus of continuity, nearly Chebyshev polynomials. 
respect to a regular (in the sense of [ST]) measure. Following [G2, we construct a sequence of "nearly Chebyshev" polynomials for $K$ and find the exact (up to a constant) value of the modulus of continuity of $g_{\mathbb{C} \backslash K}$. It should be noted that the general bound by V. Totik [T, Th. 2.2] of the Green functions, which is highly convenient to characterize optimal (that is, Lip 1/2) smoothness of $g_{\mathbb{C} \backslash K}$ for $K \subset[0,1]$, cannot be applied to our case. See Section 6 for more details.

There are several applications of smoothness properties of the Green functions to solving different problems in analysis (see e.g. [T]). We are interested in applications to polynomial inequalities. In Section 7 we evaluate the Markov factors for our set $K$.

2. Notation and the main result. Let $K=\{0\} \cup \bigcup_{k=1}^{\infty} I_{k} \subset[0,1]$, where $I_{k}=\left[a_{k}, b_{k}\right]=\left[c_{k}-\delta_{k}, c_{k}+\delta_{k}\right]$ with $a_{k} \downarrow 0$ and $h_{k}:=a_{k}-b_{k+1}>0$ for all $k$.

We use the Chebyshev polynomials $T_{n}(x)=\cos (n \cdot \arccos x)$ for $|x| \leq 1$ and $n \in \mathbb{N}_{0}:=\{0,1,2, \ldots\}$. For a fixed interval $I_{k}$, let $T_{n k}$ denote the scaled Chebyshev polynomial, that is, $T_{n k}(x)=T_{n}\left(\frac{x-c_{k}}{\delta_{k}}\right)$.

For fixed $m \in \mathbb{N}$ and $n_{k}=n_{k}(m)$ with $k=1, \ldots, m-1$, we consider the polynomial

$$
P_{N_{m}}(x)=\left(x / b_{m}\right) \prod_{k=1}^{m-1}\left[T_{n_{k} k}(x) / T_{n_{k} k}(0)\right]
$$

of degree $N_{m}=1+\sum_{k=1}^{m-1} n_{k}$. This construction appears in [G2] (see also G1]).

Here and in what follows, we adopt the convention that $\prod_{j}^{i}(\cdots)=1$ and $\sum_{j}^{i}(\cdots)=0$ for $j>i$.

We restrict our attention to the compact set $K$ with $b_{k}=\exp \left(-2^{k}\right), a_{k}=$ $b_{k}-b_{k+1}$. By Wiener's criterion ([W]), the point 0 is regular, thus $g_{\mathbb{C} \backslash K}$ is continuous throughout $\mathbb{C}$.

We find the degrees $\left(n_{k}\right)_{k=1}^{m-1}$ such that the maximal values of $\left|P_{N_{m}}(x)\right|$ for $x \in I_{k}$ are smaller than 1 for $1 \leq k \leq m-1$. Clearly, $\left|P_{N_{m}}(x)\right|<1$ for $x \leq b_{m}$. We call $P_{N_{m}}$ a nearly Chebyshev polynomial for the set $K$.

Substituting the polynomial $P_{N_{m}}$ into (1.1) at $z=-\delta$ yields a lower bound on $g_{\mathbb{C} \backslash K}(-\delta)$.

In order to get an upper bound on $g_{\mathbb{C} \backslash K}(z)$ for $z \in \mathbb{C}$ with $\operatorname{dist}(z, K)=\delta$, we fix any polynomial $P$ with $|P|_{K} \leq 1$ and, as in [AG], interpolate $P$ at zeros of a suitable nearly Chebyshev polynomial. The fundamental Lagrange polynomials are uniformly bounded by the desired value, which gives the main result. 
Let

$$
\varphi(\delta)=1 / \log (1 / \delta) \quad \text { for } 0<\delta<1, \quad \gamma=-\log (2-\sqrt{2}) / \log 2 .
$$

Theorem 2.1. Let $\operatorname{dist}(z, K)=\delta \leq b_{1}$. Then $g_{\mathbb{C} \backslash K}(z) \leq C \varphi^{\gamma}(\delta)$, where $C$ does not depend on $\delta$. On the other hand, $g_{\mathbb{C} \backslash K}(-\delta) \geq \varphi^{\gamma}(\delta)$.

We remark that the modulus of continuity of $g_{\mathbb{C} \backslash K}$ is given in terms of the function $\varphi$, which is used in the definition of the logarithmic measure (see e.g. [N, Ch. V.6]). The parameter $\gamma$ here is such that $\varphi^{\gamma}\left(b_{s}\right)=(2-\sqrt{2})^{s}$.

Given Green's function $g_{\mathbb{C} \backslash K}$, a standard application of the Cauchy formula for $P^{\prime}$ and the Bernstein-Walsh inequality allow us to estimate the Markov factors $M_{n}(K)=\sup _{P \in \mathcal{P}_{n}}\left|P^{\prime}\right|_{K} /|P|_{K}$.

COROLlary 2.2. There exists a constant $C$ such that for $n \in \mathbb{N}$ we have

$$
\exp n^{\gamma_{1}} \leq M_{n}(K) \leq \exp \left(C n^{\gamma_{1}}\right) \quad \text { with } \quad \gamma_{1}=\frac{\log 2}{\log (2+\sqrt{2})}
$$

3. Auxiliary results on Chebyshev polynomials. Let $I=[a, b]=$ $[c-\delta, c+\delta]$. We are interested in estimating the values of $T_{n I}(x)=T_{n}\left(\frac{x-c}{\delta}\right)$ for $x \notin I$.

Let $\lambda_{n}(t)=\left(1+\sqrt{1-t^{2}}\right)^{n}+\left(1-\sqrt{1-t^{2}}\right)^{n}$ for $0 \leq t \leq 1$. Then, in view of the well-known representation $T_{n}(x)=\frac{1}{2}\left[\left(x+\sqrt{x^{2}-1}\right)^{n}+\left(x-\sqrt{x^{2}-1}\right)^{n}\right]$ for $|x| \geq 1$ (see e.g. [R]), we get for $\Delta_{1}, \Delta_{2}>\delta$,

$$
\frac{\left|T_{n I}\left(c \pm \Delta_{1}\right)\right|}{\left|T_{n I}\left(c \pm \Delta_{2}\right)\right|}=\left(\frac{\Delta_{1}}{\Delta_{2}}\right)^{n} \frac{\lambda_{n}\left(\delta / \Delta_{1}\right)}{\lambda_{n}\left(\delta / \Delta_{2}\right)}
$$

where the last fraction can be estimated in the following way.

Lemma 3.1. Let $t_{1}<t_{2}<1 / 2$. Then $\lambda_{n}\left(t_{1}\right) / \lambda_{n}\left(t_{2}\right) \leq 2 \exp \left[n\left(t_{2}^{2}-t_{1}^{2}\right) / 3\right]$.

Proof. We have

$$
\begin{aligned}
\lambda_{n}\left(t_{1}\right) / \lambda_{n}\left(t_{2}\right) & <\left[\left(1+\sqrt{1-t_{1}^{2}}\right)^{n}+t_{1}^{2 n}\right] /\left(1+\sqrt{1-t_{2}^{2}}\right)^{n} \\
& =\left(1+\left(t_{2}^{2}-t_{1}^{2}\right) / a\right)^{n}+b^{n},
\end{aligned}
$$

where $a=\left(\sqrt{1-t_{1}^{2}}+\sqrt{1-t_{2}^{2}}\right)\left(1+\sqrt{1-t_{2}^{2}}\right)>3, b=t_{1}^{2} /\left(1+\sqrt{1-t_{2}^{2}}\right)$ $<1 / 7$, and the lemma follows.

Next, we will use the corresponding monic polynomial $Q_{n I}=\delta^{n} 2^{1-n} T_{n I}$, that is, $Q_{n I}(x)=\prod_{k=1}^{n}\left(x-x_{k}\right)$ with $x_{k}=c+\delta \xi_{k}$. Here, $\xi_{k}=\cos \frac{2 k-1}{2 n} \pi$ for $1 \leq k \leq n$ are the zeros of $T_{n}$. Since $T_{n}^{\prime}\left(\xi_{k}\right)=(-1)^{k-1} n / \sqrt{1-\xi_{k}^{2}}$ (see e.g. [R, 1.24]), and $n \leq\left|T_{n}^{\prime}\left(\xi_{k}\right)\right| \leq n / \sin (\pi / 2 n)<n^{2}$, we have

$$
n(\delta / 2)^{n-1} \leq\left|Q_{n I}^{\prime}\left(x_{k}\right)\right|<n^{2}(\delta / 2)^{n-1} .
$$


4. Nearly Chebyshev polynomials for $K$. The desired degrees $\left(n_{k}(m)\right)_{k=1}^{m-1}$ will be defined by means of the sequence $\left(r_{k}\right)_{k=0}^{\infty}$, where $r_{0}=r_{1}=1$ and $r_{k+1}=r_{0}+r_{1}+\cdots+r_{k-1}+3 r_{k}$ for $k \geq 1$. This gives the second-order recurrence relation $r_{k+2}=4 r_{k+1}-2 r_{k}, k \geq 1$, with the solution $r_{k}=(2 \sqrt{2})^{-1}(2+\sqrt{2})^{k}-(2 \sqrt{2})^{-1}(2-\sqrt{2})^{k}$ for $k \geq 1$. We remark that all $r_{k}$ with $k \geq 2$ are even.

Given fixed $m \in \mathbb{N}$, we define $n_{k}=r_{m-k}$ for $k=1, \ldots, m$. Thus,

$$
n_{k}=\frac{1}{2 \sqrt{2}}\left[(2+\sqrt{2})^{m-k}-(2-\sqrt{2})^{m-k}\right]
$$

for $1 \leq k \leq m-1$ and $n_{m}=1$. If $m \geq 7$ then $n_{m}, n_{m-1}, \ldots, n_{m-6}, \ldots$ are given as $1,1,4,14,48,164,560, \ldots$ If $3 \leq q \leq m-1$ then $n_{q-2}=4 n_{q-1}-2 n_{q}$. We collect together the properties of $\left(n_{k}\right)$ that will be used in what follows. Recall that $b_{k}=\exp \left(-2^{k}\right)$. The statements below follow from the definition of $\left(n_{k}\right)$ and straightforward calculations.

Lemma 4.1. Let $m \geq 3$. Then the numbers $n_{k}$ satisfy:

(1) $\sum_{k=q+1}^{m} n_{k}=n_{q-1}-3 n_{q}$ for $2 \leq q \leq m-1$,

(2) $(2 \sqrt{2})^{-1}(2+\sqrt{2})^{m-k}-1<n_{k}<(2 \sqrt{2})^{-1}(2+\sqrt{2})^{m-k}$ for $1 \leq k \leq$ $m-1$,

(3) $4=n_{m-2} / n_{m-1}>n_{m-3} / n_{m-2}>\cdots>n_{1} / n_{2}>2+\sqrt{2}$,

(4) $b_{m} \cdot b_{m-1} \cdot b_{m-2}^{4} \cdots b_{q+1}^{n_{q+1}}=b_{q}^{1+1+4+\cdots+n_{q}}$ for $1 \leq q \leq m-1$,

(5) $N_{m}=\sum_{k=1}^{m} n_{k}=\frac{1}{2}\left[(2+\sqrt{2})^{m-1}+(2-\sqrt{2})^{m-1}\right]<(2+\sqrt{2}) N_{m-1}$,

(6) $n_{k} / N_{m}<(1+\sqrt{2}) 2^{-k}(2-\sqrt{2})^{k}=(1+\sqrt{2})(2+\sqrt{2})^{-k}$ for $1 \leq k \leq$ $m-1$

(7) $\sum_{k=p}^{q} n_{k}<\sqrt{2} n_{p}$ for $1 \leq p \leq q \leq m-1$,

(8) $\sum_{k=p}^{q} n_{k} b_{k}<2 n_{p} b_{p}$ and $\sum_{k=p}^{q} n_{k} / b_{k}<2 n_{q} / b_{q}$ for $1 \leq p \leq q \leq m$.

Suppose the polynomial $P_{N_{m}}$ is defined by means of $\left(n_{k}\right)_{k=1}^{m-1}$ and the compact set $K$ is given as in Section 2 .

Lemma 4.2. Given $m \in \mathbb{N}$, let $\left(n_{k}\right)_{k=1}^{m-1}$ be defined as above. Then $\left|P_{N_{m}}(x)\right|$ $\leq 1$ for $x \in K$.

Proof. The result is evident for $P_{1}(x)=x / b_{1}$ and $P_{2}(x)=\left(x / b_{2}\right)$. $\left(c_{1}-x\right) / c_{1}$. Hence we can suppose that $m \geq 3$ and use Lemma 4.1.

Fix $x \in K$. If $x \leq b_{m}$ then $0<T_{n_{k} k}(x) / T_{n_{k} k}(0) \leq 1$ for all $k \leq m-1$, so $\left|P_{N}(x)\right| \leq 1$.

Suppose $x \in I_{q}$ with $1 \leq q \leq m-2$. Then $0<T_{n_{k} k}(x) / T_{n_{k} k}(0) \leq 1$ for $1 \leq k \leq q-1$ and $\left|T_{n_{q} q}(x)\right| \leq 1$. Therefore, we need to check

$$
\left(b_{q} / b_{m}\right) \prod_{k=q+1}^{m-1}\left|T_{n_{k} k}\left(b_{q}\right) / T_{n_{k} k}(0)\right| \leq\left|T_{n_{q} q}(0)\right| .
$$


From (3.1) we have

$$
\begin{aligned}
\left|T_{n_{k} k}\left(b_{q}\right) / T_{n_{k} k}(0)\right|=\left(\frac{b_{q}-c_{k}}{c_{k}}\right)^{n_{k}} \frac{\lambda_{n_{k}}\left(t_{1}\right)}{\lambda_{n_{k}}\left(t_{2}\right)} & \text { with } \\
t_{1} & =\frac{b_{k+1}}{2\left(b_{q}-c_{k}\right)}<t_{2}=\frac{b_{k+1}}{2 c_{k}} .
\end{aligned}
$$

Here, $c_{k}=b_{k}-\frac{1}{2} b_{k+1}$, so $\left(b_{q}-c_{k}\right) / c_{k}<b_{q} / b_{k}$ and, by Lemma 3.1, $\lambda_{n_{k}}\left(t_{1}\right) / \lambda_{n_{k}}\left(t_{2}\right)<2 \exp \left(n_{k} t_{2}^{2} / 3\right)<2 \exp \left(n_{k} b_{k}\right)$. Therefore the left side in (4.1) does not exceed

$$
\frac{b_{q}^{n_{m}+n_{m-1}+\cdots+n_{q+1}}}{b_{m} b_{m-1} b_{m-2}^{n_{m-2}} \cdots b_{q+1}^{n_{q+1}}} 2^{m-q-1} \exp \left(\sum_{k=q+1}^{m-1} n_{k} b_{k}\right) .
$$

Lemma 4.1(4)\&(8) now shows that the first fraction above is $b_{q}^{-n_{q}}$ and $\sum_{k=q+1}^{m-1} n_{k} b_{k}<2 n_{q+1} b_{q+1}$, so the product is less than $b_{q}^{-n_{q}} 2^{m-q-1} e^{2 n_{q+1} b_{q+1}}$.

On the other hand, the right side of (4.1) is $\frac{1}{2}\left(\frac{c_{q}}{b_{q+1} / 2}\right)^{n_{q}} \lambda_{n_{q}}\left(\frac{b_{q+1}}{2 c_{q}}\right)$. Clearly, $\lambda_{n}(t) \geq 2$ and $2 c_{q} / b_{q+1}=\left(2-b_{q}\right) / b_{q}$. It follows that $\left|T_{n_{q} q}(0)\right| \geq b_{q}^{-n_{q}}\left(2-b_{q}\right)^{n_{q}}$ and we only need to show that

$$
(m-q-1) \log 2+2 n_{q+1} b_{q+1}<n_{q} \log \left(2-b_{q}\right) .
$$

By Lemma 4.1(3), this can be reduced to $2(m-q-1) \log 2<n_{q}$, which is easy to check.

In the last case, when $x \in I_{m-1}$, the condition (4.1) assumes the form

$$
b_{m-1} / b_{m} \leq\left|T_{1, m-1}(0)\right|=2 c_{m-1} / b_{m},
$$

which evidently is fulfilled.

5. Proof of the main result. Let us first prove the simpler sharpness result. Fix $\delta \leq b_{1}$ and $s \geq 1$ with $b_{s+1}<\delta \leq b_{s}$. We consider the polynomial $P=P_{N_{s+2}}$, that is, $P(x)=\left(x / b_{s+2}\right) \prod_{k=1}^{s+1}\left[T_{n_{k} k}(x) / T_{n_{k} k}(0)\right]$. By (1.1) and Lemma 4.2, we have $g_{\mathbb{C} \backslash K}(-\delta) \geq N^{-1} \log |P(-\delta)|$. Here, $N=\frac{1}{2}(2+\sqrt{2})^{s+1}\left[1+(\sqrt{2}-1)^{2 s+2}\right]$, by Lemma 4.1(5). Since $\left|T_{n_{k} k}(-\delta)\right|>$ $\left|T_{n_{k} k}(0)\right|$, we see that $|P(-\delta)|>\delta / b_{s+2}>b_{s+1}^{-1}$ and

$$
g_{\mathbb{C} \backslash K}(-\delta)>\frac{2^{s+1}}{N}=\frac{4}{(2+\sqrt{2})\left[1+(\sqrt{2}-1)^{2 s+2}\right]}\left(\frac{2}{2+\sqrt{2}}\right)^{s} .
$$

The first fraction exceeds 1 and $\left(\frac{2}{2+\sqrt{2}}\right)^{s}=(2-\sqrt{2})^{s}=\varphi^{\gamma}\left(b_{s}\right)$, thus $g_{\mathbb{C} \backslash K}(-\delta)>\varphi^{\gamma}\left(b_{s}\right) \geq \varphi^{\gamma}(\delta)$, which is the desired conclusion.

We proceed to estimate $g_{\mathbb{C} \backslash K}$ from above. Let us first prove that

$$
g_{\mathbb{C} \backslash K}\left(-b_{s}\right) \leq C(2-\sqrt{2})^{s}
$$


for $s \geq 2$, where $C$ does not depend on $s$. In order to show this, we will define a certain increasing sequence $\left(D_{m}\right)$ with

$$
D_{m}<N_{m}<12 D_{m},
$$

where $N_{m}$ is given in Lemma 4.1(5). For each large $m$ we will consider a system $\left(x_{k}\right)_{k=1}^{D_{m}}$ of interpolating points on $K$. Then any polynomial $P_{N}$ with $D_{m-1} \leq N<D_{m}$ can be represented in the form $P_{N}=\sum_{k=1}^{D_{m}} P_{N}\left(x_{k}\right) L_{k}$, where $\left(L_{k}\right)_{k=1}^{D_{m}}$ are the corresponding Lagrange fundamental polynomials. We will show that for $s \geq 2$,

$$
\left(\log \left|L_{k}\left(-b_{s}\right)\right|\right) / N_{m} \leq C_{1}(2-\sqrt{2})^{s},
$$

where $C_{1}$ does not depend on $s$ or $k$.

Suppose $\left|P_{N}\right|_{K} \leq 1$. Then $\left|P_{N}\left(-b_{s}\right)\right| \leq N_{m} \max _{1 \leq k \leq D_{m}}\left|L_{k}\left(-b_{s}\right)\right|$. By (5.2) and Lemma 4.1(5), we get $N_{m}<12(2+\sqrt{2}) N=C_{2} N$. Therefore,

$$
\left(\log \left|P_{N}\left(-b_{s}\right)\right|\right) / N \leq \log \left(C_{2} N\right) / N+C_{1} C_{2}(2-\sqrt{2})^{s} .
$$

Since in the representation (1.1) we can consider only polynomials of arbitrarily large degrees, the second term in the sum above dominates, which establishes the desired result (5.1).

We proceed to define the numbers $\left(D_{m}\right)$ and the corresponding interpolating points. Given $s \geq 2$, fix $m \geq s+2$. Let $\left(n_{q}\right)_{q=1}^{m}$ and $P_{N_{m}}$ be defined as above. The bound (5.3) is not valid if we use the zeros of $P_{N_{m}}$ as interpolating points. For this reason we introduce new degrees $d_{q}=n_{q}-\nu_{q}$ by means of the correction terms $\nu_{q}=\left[n_{q} 2^{-q} \log 8\right]$, where $[x]$ denotes the greatest integer less than or equal to $x$. We remark that $d_{q}=n_{q}$ for large $q$, namely $q=m, m-1, \ldots, m_{1}$, with $m_{1} \approx m \cdot \log (2+\sqrt{2}) / \log (4+\sqrt{2})$, whereas for small values of $q$ the correction is essential. Since $\nu_{1}>n_{1}$, we take $d_{1}=0$. An easy computation shows that

$$
8^{-n_{q}} \leq b_{q}^{\nu_{q}}<8^{-n_{q}} b_{q}^{-1} \quad \text { for all } q .
$$

Let us estimate the sum $\sum_{k=q}^{m} \nu_{k}$ from above, where $q \geq 2$ and the actual summation is only till $m_{1}$, in view of the previous remark. By Lemma 4.1(2),

$$
\sum_{k=q}^{m} \nu_{k} \leq \frac{\log 8}{2 \sqrt{2}} \sum_{k=q}^{m} \frac{(2+\sqrt{2})^{m-k}}{2^{k}}<\frac{\log 8}{2 \sqrt{2}} \frac{(2+\sqrt{2})^{m-q}}{2^{q}} \sum_{k=0}^{\infty}(4+2 \sqrt{2})^{-k} .
$$

We will denote the last sum by $\rho$, so $\rho=(4+2 \sqrt{2}) /(3+2 \sqrt{2})$. On the other hand, the lower bound of $n_{k}$ in Lemma 4.1(2) implies

$$
\nu_{q}>\frac{\log 8}{2^{q}}\left[\frac{1}{2 \sqrt{2}}(2+\sqrt{2})^{m-q}-1\right]-1 .
$$


Thus,

$$
\sum_{k=q}^{m} \nu_{k}<\rho \nu_{q}+\rho\left(2^{-q} \log 8+1\right)<\rho \nu_{q}+2,
$$

since $\rho<6 / 5$ and $q \geq 2$.

Let us take $x_{1}=0, x_{2}=b_{m}$ and then $d_{q}$ Chebyshev points on each interval $I_{q}$ with $q=m-1, m-2, \ldots, 2$. That is, $x_{3}=c_{m-1}$, since $d_{m-1}=$ $n_{m-1}=1$ and $T_{1, m-1}(x)=2\left(x-c_{m-1}\right) / b_{m}$. Then $x_{4}, \ldots, x_{7}$ are the zeros of $T_{d_{m-2}, m-2}$, etc. Thus, $D_{m}:=1+\sum_{q=2}^{m} d_{q}$ is the total number of interpolating points for given $m$.

We proceed to show that (5.5) implies (5.2). Clearly, $D_{m}=1+$ $\sum_{q=2}^{m}\left(n_{q}-\nu_{q}\right)$ does not exceed $N_{m}=\sum_{q=1}^{m} n_{q}$. The second inequality in (5.2) is equivalent to $N_{m}<12\left(1+N_{m}-n_{1}-\sum_{k=2}^{m} \nu_{k}\right)$, which can be reduced, by (5.5), to $12 \rho \nu_{2}+12+12 n_{1}<11 N_{m}$. Here, $\nu_{2} \leq\left(n_{2} / 4\right) \log 8$ and, by Lemma 4.1(6), $N_{m}>\sqrt{2} n_{1}$. Hence, it is enough to show that $3 \rho n_{2} \log 8+12<(11 \sqrt{2}-12) n_{1}$. Since, by Lemma $4.1(3), n_{1}>n_{2}(2+\sqrt{2})$, we need to check that $12<(10 \sqrt{2}-2-3 \rho \log 8) n_{2}$. Recall that $s \geq 2$ and $m \geq s+2$, so $n_{2} \geq n_{m-2}=4$. Finally, the inequality $5+3 \rho \log 8<10 \sqrt{2}$ is valid for $\rho<6 / 5$.

Our next goal is to prove (5.3). To shorten notation we write $Q_{j}$ for the monic Chebyshev polynomial $Q_{d_{j}, j}$ of degree $d_{j}$ on $I_{j}$, and $t_{j}:=$ $\left|Q_{j}\left(-b_{s}\right) / Q_{j}\left(x_{k}\right)\right|$.

Suppose first $x_{k} \in I_{q}$ with $s \leq q \leq m-1$. Then $\left|L_{k}\left(-b_{s}\right)\right|=\pi_{1} \pi_{2} \pi_{3} \pi_{4}$, where

$$
\pi_{1}=\frac{b_{s}\left(b_{s}+b_{m}\right)}{x_{k}\left(x_{k}-b_{m}\right)} \prod_{j=q+1}^{m-1} t_{j}, \pi_{2}=\frac{\left|Q_{q}\left(-b_{s}\right)\right|}{\left(b_{s}+x_{k}\right)\left|Q_{q}^{\prime}\left(x_{k}\right)\right|}, \pi_{3}=\prod_{j=s}^{q-1} t_{j}, \pi_{4}=\prod_{j=2}^{s-1} t_{j} .
$$

For the terms in the product $\pi_{1}$ we have $\left|Q_{j}\left(-b_{s}\right)\right|<b_{s}^{d_{j}}\left(1+b_{j} / b_{s}\right)^{d_{j}}$ and $\left|Q_{j}\left(x_{k}\right)\right|>b_{q}^{d_{j}}\left(1-b_{q}-b_{j} / b_{q}\right)^{d_{j}}$. Therefore, $\pi_{1}<\left(b_{s} / b_{q}\right)^{1+d_{m}+\cdots+d_{q+1}} A_{1} / B_{1}$, where $A_{1}=\prod_{j=q+1}^{m}\left(1+b_{j} / b_{s}\right)^{d_{j}} \leq \prod_{j=q+1}^{m}\left(1+b_{j} / b_{s}\right)^{n_{j}}<e^{2 n_{q+1} b_{q}}$, by Lemma 4.1(8). Similarly, $B_{1}=\left(1-b_{q}\right) \prod_{j=q+1}^{m}\left(1-b_{q}-b_{j} / b_{q}\right)^{d_{j}}$. Since $b_{j} / b_{q} \leq b_{q}$ and $d_{j} \leq n_{i}$, we have, by Lemma 4.1(1),

$$
B_{1}>\left(1-2 b_{q}\right)^{1+n_{m}+\cdots+n_{q+1}}=\left(1-2 b_{q}\right)^{1+n_{q-1}-3 n_{q}}>\left(1-2 b_{q}\right)^{n_{q-1}} .
$$

Hence, $B_{1}>\left(1+3 b_{q}\right)^{-n_{q-1}}$ and $B_{1}>e^{-3 n_{q-1} b_{q}}$. We can replace $d_{j}$ by $n_{j}$ also in the exponent of $b_{s} / b_{q}$. Lemma 4.1(1) now yields

$$
\log \pi_{1}<\left(n_{q-1}-3 n_{q}+1\right)\left(2^{q}-2^{s}\right)+b_{q}\left(2 n_{q+1}+3 n_{q-1}\right) .
$$

In addition, $n_{q-1} \leq 4 n_{q}$ and $q \geq s \geq 2$. Therefore, $\left(n_{q-1}-3 n_{q}+1\right) 2^{q} \leq$ $n_{q} 2^{q+1}$ and $b_{q}\left(2 n_{q+1}+3 n_{q-1}\right)<4 n_{q} \leq 2^{s} n_{q}$. Thus, $\log \pi_{1}<n_{q} 2^{q+1}$ and, by 
Lemma 4.1(6),

$$
\left(\log \pi_{1}\right) / N_{m}<2(1+\sqrt{2})(2-\sqrt{2})^{q} \leq(2+2 \sqrt{2})(2-\sqrt{2})^{s} .
$$

Let us estimate $\pi_{2}$ from above. The value $\left|Q_{q}\left(-b_{s}\right)\right|$ consists of $d_{q}$ terms. One of them coincides with $b_{s}+x_{k}$. Hence, $\left|Q_{q}\left(-b_{s}\right)\right| /\left(b_{s}+x_{k}\right)<\left(b_{s}+b_{q}\right)^{d_{q}-1}$ $\leq\left(2 b_{s}\right)^{d_{q}-1}$, as $s \leq q$. On the other hand, by (3.2), $\left|Q_{q}^{\prime}\left(x_{k}\right)\right|>d_{q}\left(b_{q+1} / 4\right)^{d_{q}-1}$. Therefore,

$$
\pi_{2}<\left(8 b_{s} / b_{q+1}\right)^{d_{q}-1}<b_{q+1}^{-d_{q}+1}<b_{q+1}^{-n_{q}}=\exp \left(n_{q} 2^{q+1}\right) .
$$

Hence $\left(\log \pi_{2}\right) / N_{m}$ has the desired bound.

Arguing as above, we see that

$$
\pi_{3}<\frac{b_{s}^{n_{q-1}+\cdots+n_{s}} 2^{n_{s}}}{b_{q-1}^{n_{q-1}} \cdots b_{s}^{n_{s}}} \frac{A_{3}}{B_{3}} \leq \frac{b_{s}^{n_{s+1}} 2^{n_{s}} A_{3}}{b_{q-1}^{n_{q-1}} \cdots b_{s+1}^{n_{s+1}} B_{3}}
$$

with $A_{3}=\prod_{j=s+1}^{q-1}\left(1+b_{j} / b_{s}\right)^{n_{j}}$ and $B_{3}=\prod_{j=s}^{q-1}\left(1-2 b_{j}\right)^{n_{j}}$. An easy computation shows that $\log \left(A_{3} / B_{3}\right) \leq 2 b_{s}\left(n_{s+1}+3 n_{s}\right)$ and

$$
b_{s}^{n_{s+1}} 2^{n_{s}} A_{3}<B_{3}
$$

for $s \geq 2$. Thus, $\pi_{3}<\left(b_{q-1}^{n_{q-1}} \cdots b_{s+1}^{n_{s+1}}\right)^{-1}$ and $\log \pi_{3}<\sum_{j=s+1}^{q-1} n_{j} 2^{j}$, so, by Lemma 4.1(6),

$$
\left(\log \pi_{3}\right) / N_{m}<(1+\sqrt{2}) \sum_{j=s+1}^{q-1}(2-\sqrt{2})^{j}<(2+\sqrt{2})(2-\sqrt{2})^{s} .
$$

To deal with $\pi_{4}$, we use (3.1):

$$
\pi_{4}=\prod_{j=2}^{s-1}\left(\frac{c_{j}+b_{s}}{c_{j}-x_{k}}\right)^{d_{j}} \frac{\lambda_{d_{j}}\left(t_{1}\right)}{\lambda_{d_{j}}\left(t_{2}\right)}
$$

with $t_{1}=\frac{b_{j+1}}{2\left(c_{j}+b_{s}\right)}$ and $t_{2}=\frac{b_{j+1}}{2\left(c_{j}-x_{k}\right)}$. Here, $t_{2}^{2}-t_{1}^{2}<2 b_{s} b_{j}$. Hence, by Lemmas 3.1 and $4.1(8)$,

$$
\prod_{j=2}^{s-1} \frac{\lambda_{d_{j}}\left(t_{1}\right)}{\lambda_{d_{j}}\left(t_{2}\right)}<2^{s-2} \exp \left(2 b_{s} n_{2} b_{2}\right) .
$$

On the other hand, $\frac{c_{j}+b_{s}}{c_{j}-x_{k}}<1+\frac{3 b_{s}}{b_{j}}$. From this,

$$
\prod_{j=2}^{s-1}\left(\frac{c_{j}+b_{s}}{c_{j}-x_{k}}\right)^{d_{j}}<\exp \left(3 b_{s} \sum_{j=2}^{s-1} n_{j} / b_{j}\right)<\exp \left(6 n_{s-1} b_{s-1}\right) .
$$

This and Lemma 4.1(6) imply that

$\left(\log \pi_{4}\right) / N_{m}$

$$
<(s-2) \log 2 / N_{m}+2 b_{s} b_{2}(1+\sqrt{2}) 2^{-2}(2-\sqrt{2})^{2}+6 b_{s-1}(1+\sqrt{2})(2+\sqrt{2})^{1-s} .
$$


Since $N_{m}>(2+\sqrt{2})^{m-1} / 2$, the first term in the sum above does not exceed $(2-\sqrt{2})^{s}$. The same bound is valid for the second term, as $1+\sqrt{2}<$ $2(2-\sqrt{2})^{s-2} e^{4} e^{2^{s}}$. For the last term we have $6 b_{s-1}(1+\sqrt{2})(2+\sqrt{2})^{1-s}<$ $2(2-\sqrt{2})^{s}$, since $3(4+3 \sqrt{2})<2^{s} e^{2^{s-1}}$ for $s \geq 2$. Therefore,

$$
\left(\log \pi_{4}\right) / N_{m}<4(2-\sqrt{2})^{s},
$$

which is the desired conclusion.

Combining these we get (5.3) for $x_{k} \in I_{q}$ with indicated values of $q$. We note that above we did not use the difference between $d_{j}$ and $n_{j}$.

The cases $k=1$ and $k=2$ are simpler and very similar. For $x_{1}=0$ we have

$$
\left|L_{1}\left(-b_{s}\right)\right|=\left(\frac{b_{s}+b_{m}}{b_{m}} \prod_{j=s}^{m-1} t_{j}\right)\left(\prod_{j=2}^{s-1} t_{j}\right)=\pi_{3} \pi_{4}
$$

with $t_{j}:=\left|Q_{j}\left(-b_{s}\right) / Q_{j}(0)\right|$. We denote the corresponding parts of the product above by $\pi_{3}$ and $\pi_{4}$ because they are handled in the same way as $\pi_{3}$ and $\pi_{4}$ in the general case. Now,

$$
\pi_{3}<\frac{b_{s}^{n_{m}+\cdots+n_{s+1}}}{b_{m}^{n_{m}} \cdots b_{s+1}^{n_{s+1}}} 2^{n_{s}} \frac{A_{3}}{B_{3}}
$$

with $A_{3}=\prod_{j=s+1}^{m}\left(1+b_{j} / b_{s}\right)^{n_{j}}$ and $B_{3}=\prod_{j=s}^{m}\left(1-b_{j}\right)^{n_{j}}$, so we can use the previous bound for $A_{3} / B_{3}$ and (5.5). Therefore, $\pi_{3}<\left(b_{m}^{n_{m}} \cdots b_{s+1}^{n_{s+1}}\right)^{-1}$ and the bound (5.7) is valid in this case as well.

Likewise, the value $\pi_{4}$ is the same as above if we take $x_{k}=0$.

The same reasoning, with a minor modification of $A_{3}$ and $B_{3}$, applies to the case $k=2$.

It remains to consider the most difficult case $x_{k} \in I_{q}$ with $2 \leq q \leq s-1$. Recall that $d_{1}=0$, so the interval $I_{1}$ does not contain interpolating points. Now we use the decomposition $\left|L_{k}\left(-b_{s}\right)\right|=\pi_{1} \pi_{2} \pi_{3} \pi_{4}$ with

$$
\pi_{1}=\frac{b_{s}\left(b_{s}+b_{m}\right)}{x_{k}\left(x_{k}-b_{m}\right)} \prod_{j=s}^{m-1} t_{j}, \pi_{2}=\prod_{j=q+1}^{s-1} t_{j}, \pi_{3}=\frac{\left|Q_{q}\left(-b_{s}\right)\right|}{\left(b_{s}+x_{k}\right)\left|Q_{q}^{\prime}\left(x_{k}\right)\right|}, \pi_{4}=\prod_{j=2}^{q-1} t_{j},
$$

where, as above, $t_{j}$ means $\left|Q_{j}\left(-b_{s}\right) / Q_{j}\left(x_{k}\right)\right|$. We note that $\pi_{4}=1$ for $q=2$.

As before, $\pi_{1}<\left(b_{s} / b_{q}\right)^{1+d_{m}+\cdots+d_{s}} 2^{d_{s}} A_{1} / B_{1}$ with $A_{1}=\prod_{j=s+1}^{m}\left(1+b_{j} / b_{s}\right)^{d_{j}}$ $<\exp \left(2 b_{s} n_{s+1}\right), B_{1}=\left(1-b_{q}\right) \prod_{j=s}^{m}\left(1-b_{q}-b_{j} / b_{q}\right)^{d_{j}}>\exp \left(-3 b_{q} n_{s}\right)$. Since, by Lemma $4.1(3), n_{s} \log 2+2 b_{s} n_{s+1}+3 b_{q} n_{s}<n_{s}$, we get

$$
\pi_{1}<\left(b_{s} / b_{q}\right)^{1+d_{m}+\cdots+d_{s}} e^{n_{s}} .
$$

If $q+1 \leq j \leq s-1$, then $b_{j}$ dominates $b_{s}$. Therefore,

$$
\pi_{2}<\frac{b_{s-1}^{d_{s-1}} \cdots b_{q+1}^{d_{q+1}}}{b_{q}^{d_{s-1}+\cdots+d_{q+1}}} \frac{A_{2}}{B_{2}}
$$


with $A_{2}=\prod_{j=q+1}^{s-1}\left(1+b_{s} / b_{j}\right)^{n_{j}}<\exp \left(2 b_{s-1} n_{s-1}\right), B_{2}=\prod_{j=q+1}^{s-1}\left(1-2 b_{q}\right)^{n_{j}}$. Here, $\left(1-2 b_{q}\right)^{-1}<1+3 b_{q}$. It follows that $B_{2}>\exp \left(-3 b_{q} \sum_{j=q+1}^{s-1} n_{j}\right)>$ $\exp \left(-3 \sqrt{2} b_{q} n_{q+1}\right)$, by Lemma 4.1(7). Combining these we get

$$
\pi_{2}<\frac{b_{s-1}^{d_{s-1}} \cdots b_{q+1}^{d_{q+1}}}{b_{q}^{d_{s-1}+\cdots+d_{q+1}}} e^{2 b_{s-1} n_{s-1}+3 \sqrt{2} b_{q} n_{q+1}} .
$$

From (3.2) we obtain

$$
\pi_{3}<\frac{\left(b_{q}+b_{s}\right)^{d_{q}-1}}{d_{q}\left(b_{q+1} / 4\right)^{d_{q}-1}}=\frac{1}{d_{q}}\left(\frac{4}{b_{q}}\right)^{d_{q}-1}\left(1+\frac{b_{s}}{b_{q}}\right)^{d_{q}-1}<\frac{1}{d_{q}}\left(\frac{4}{b_{q}}\right)^{d_{q}-1} e^{n_{s} b_{q}} .
$$

Similarly to the case $s \leq q$, for $\pi_{4}$ we use (3.1):

$$
\pi_{4}=\prod_{j=2}^{q-1}\left(\frac{c_{j}+b_{s}}{c_{j}-x_{k}}\right)^{d_{j}} \frac{\lambda_{d_{j}}\left(t_{1}\right)}{\lambda_{d_{j}}\left(t_{2}\right)} \quad \text { with } \quad t_{1}=\frac{b_{j+1}}{2\left(c_{j}+b_{s}\right)}, t_{2}=\frac{b_{j+1}}{2\left(c_{j}-x_{k}\right)} .
$$

As above,

$$
t_{2}^{2}-t_{1}^{2}=\frac{b_{j+1}^{2}}{4} \frac{\left(b_{s}+x_{k}\right)\left(2 c_{j}+b_{s}-x_{k}\right)}{\left(c_{j}+b_{s}\right)^{2}\left(c_{j}-x_{k}\right)^{2}} .
$$

Here, $b_{s}+x_{k}<2 b_{q}, 2 c_{j}+b_{s}-x_{k}<2\left(c_{j}+b_{s}\right)$, and $\left(c_{j}+b_{s}\right)\left(c_{j}-x_{k}\right)^{2}>b_{j}^{3} / 2$. Hence, $t_{2}^{2}-t_{1}^{2}<2 b_{q} b_{j}$. By Lemmas 3.1 and $4.1(8)$,

$$
\prod_{j=2}^{q-1} \frac{\lambda_{d_{j}}\left(t_{1}\right)}{\lambda_{d_{j}}\left(t_{2}\right)}<2^{q} \exp \left(\frac{4}{3} b_{q} n_{2} b_{2}\right) \text {. }
$$

Also,

$$
\frac{c_{j}+b_{s}}{c_{j}-x_{k}}<1+\frac{2 b_{q}}{b_{j}} \text { and } \prod_{j=2}^{q-1}\left(\frac{c_{j}+b_{s}}{c_{j}-x_{k}}\right)^{d_{j}}<\exp \left(4 n_{q-1} b_{q-1}\right) .
$$

Therefore,

$$
\pi_{4}<\exp \left(4 n_{q-1} b_{q-1}+q+\frac{4}{3} b_{q} n_{2} b_{2}\right) \quad \text { for } q \geq 3 .
$$

Combining all inequalities yields

$$
\begin{aligned}
& \left|L_{k}\left(-b_{s}\right)\right| \\
& \quad<\frac{b_{s}^{1+n_{m}+\cdots+n_{s}} b_{s-1}^{n_{s-1}} \cdots b_{q+1}^{n_{q+1}}}{b_{q}^{1+n_{m}+\cdots+n_{q+1}} b_{q}^{n_{q}-1}} \frac{b_{q}^{\nu_{q}+\nu_{q+1}+\cdots}}{b_{s}^{\nu_{s}+\nu_{s+1}+\cdots} b_{s-1}^{\nu_{s-1}} \cdots b_{q+1}^{\nu_{q+1}}} \frac{4^{n_{q}-\nu_{q}-1}}{d_{q}} e^{\mu_{1}+\mu_{2}},
\end{aligned}
$$

where $\mu_{1}=n_{s}+2 b_{s-1} n_{s-1}+n_{s} b_{q}+q<2 n_{s}+q, \mu_{2}=3 \sqrt{2} b_{q} n_{q+1}+4 n_{q-1} b_{q-1}+$ $\frac{4}{3} b_{q} n_{2} b_{2}$ and $\nu_{i}+\nu_{i+1}+\cdots$ denotes the sum of all nonzero correction terms starting from $\nu_{i}$.

Let us consider the first fraction in the product above. By Lemma 4.1(4), $b_{s}^{\sum_{k=s}^{m} n_{s}}=\prod_{k=s+1}^{m} b_{k}^{n_{k}}$. Therefore the numerator of this fraction equals 
$b_{s}^{1-n_{s}} \prod_{k=q+1}^{m} b_{k}^{n_{k}}$. On the other hand, its denominator is just $\prod_{k=q+1}^{m} b_{k}^{n_{k}}$, for the same reason. Hence the first fraction above is $b_{s}^{1-n_{s}}$.

Further, $b_{q}^{\nu_{q}+\nu_{q+1}+\cdots} \leq b_{q}^{\nu_{q}+\nu_{q+1}}=b_{q}^{\nu_{q}} b_{q+1}^{\nu_{q+1} / 2}<8^{-n_{q}-n_{q+1} / 2} b_{q}^{-2}$, by (5.4). In turn, by (5.5), the denominator of the second fraction is larger than $b_{s}^{\rho \cdot \nu_{s}+2} 8^{-n_{s-1}-\cdots-n_{q+1}}$. Thus the second fraction does not exceed $b_{q}^{-2} b_{s}^{-2} 8^{-\kappa}$, where $\kappa=n_{q}+\frac{1}{2} n_{q+1}-\left(n_{q+1}+\cdots+n_{s-1}+\rho \cdot n_{s}\right)$. Here, $\rho n_{s}<n_{s}+n_{s+1}$. Hence, by Lemma 4.1(7),

$$
\kappa>n_{q}-\left(\sqrt{2}-\frac{1}{2}\right) n_{q+1}>\frac{5}{2(2+\sqrt{2})} n_{q}>\frac{2}{3} n_{q} .
$$

Therefore,

$$
\left|L_{k}\left(-b_{s}\right)\right|<b_{s}^{-1-n_{s}} b_{q}^{-2} e^{2 n_{s}+q} \frac{4^{n_{q}}}{8^{\kappa}} \frac{e^{\mu_{2}}}{4^{\nu_{q}}} .
$$

From a computational point of view, we introduce the correction terms $\left(\nu_{q}\right)$ in order to neutralize $4^{n_{q}}$ in the numerator above, which is unacceptably large if we use the degrees $\left(n_{q}\right)$ without correction.

According to the estimation of $\kappa$, we have $4^{n_{q}}<8^{\kappa}$. Let us show that

$$
e^{\mu_{2}} \leq 4^{\nu_{q}+1} \text {. }
$$

If $q=2$ then $\pi_{4}=1$ and $\mu_{2}$ contains only its first term, that is, $\mu_{2}=3 \sqrt{2} b_{2} n_{3}$. By Lemma 4.1(3), $\mu_{2}<\frac{3 \sqrt{2}}{2+\sqrt{2}} e^{-4} n_{2}$. On the other hand, $\left(\nu_{2}+1\right) \log 4>\frac{n_{2}}{4} 6 \log ^{2} 2$, which exceeds $\mu_{2}$.

Thus we can suppose that $q \geq 3$. Since $q \leq s-1$ and $m \geq s+2$, we have $\frac{n_{q-1}}{n_{q}} \leq \frac{n_{m-4}}{n_{m-3}}<\frac{7}{2}$, by Lemma $4.1(3)$. Also, $n_{2}<2(2+\sqrt{2})^{q-2} n_{q}$, by Lemma 4.1(2). Therefore,

$$
\mu_{2}<n_{q}\left[\frac{3 \sqrt{2}}{2+\sqrt{2}} b_{q}+14 b_{q-1}+\frac{8}{3}(2+\sqrt{2})^{q-2} b_{q} b_{2}\right] .
$$

On the other hand, $\left(\nu_{q}+1\right) \log 4>\frac{n_{q}}{2^{q}} 6 \log ^{2} 2$. It is enough to show that

$$
2^{q}\left[\frac{3 \sqrt{2}}{2+\sqrt{2}} e^{-2^{q}}+14 e^{-2^{q-1}}+\frac{8}{3}(2+\sqrt{2})^{q-2} e^{-2^{q}} e^{-4}\right]<6 \log ^{2} 2 .
$$

The expression on the left attains its maximal value at the minimal $q$, so we reduced the proof to the case $q=3$, which can be checked by a straightforward calculation.

From this,

$\log \left|L_{k}\left(-b_{s}\right)\right|<\left(n_{s}+1\right) 2^{s}+2^{q+1}+2 n_{s}+q+2 \leq n_{s}\left(2^{s}+2\right)+2^{s+1}+s+1$, since $q+1 \leq s$. Recall that $m \geq s+2$, so $n_{s} \geq n_{m-2}=4$. Therefore, $\log \left|L_{k}\left(-b_{s}\right)\right|<n_{s} 2^{s+2}$, which gives (5.3) in view of Lemma 4.1(6). This completes the proof of (5.1). 
We now turn to the general case. Suppose $\operatorname{dist}(z, K)=\delta \leq b_{1}$. We want to show that $g_{\mathbb{C} \backslash K}(z) \leq C \varphi^{\gamma}(\delta)$, where $C$ does not depend on $\delta$. We can assume, by increasing $C$ if necessary, that $\delta \leq b_{s_{0}}$ for any $s_{0}$ given beforehand. Take $s_{0}=4$. Fix $z$ with $\operatorname{dist}(z, K)=\delta \leq b_{4}$ and $s \geq 4$ such that $b_{s+1}<\delta \leq b_{s}$. Since $\frac{1}{2} \varphi\left(b_{s}\right)=\varphi\left(b_{s+1}\right)<\varphi(\delta) \leq \varphi\left(b_{s}\right)$, it is enough to show that

$$
g_{\mathbb{C} \backslash K}(z) \leq C(2-\sqrt{2})^{s}
$$

for $z$ with $\operatorname{dist}(z, K)=b_{s}$, where $C$ does not depend on $s$.

Suppose first that $\operatorname{dist}(z, K)=\left|z-z_{0}\right|$ with $z_{0} \in I_{q}$ for some $q$ with $q \leq s-2$. The monotonicity of the Green function with respect to the set $K$ implies that $g_{\mathbb{C} \backslash K}(z) \leq g_{\mathbb{C} \backslash I_{q}}(z)$. It is well-known that, given $I=[-l, l]$, the Green function $g_{\mathbb{C} \backslash I}(z)=\log \left|z / l+\sqrt{(z / l)^{2}-1}\right|$ attains its maximal value, among all $z$ with $\operatorname{dist}(z, I)=\delta$, at real points. Therefore,

$$
\max \left\{g_{\mathbb{C} \backslash I}(z): \operatorname{dist}(z, I)=\delta\right\}=g_{\mathbb{C} \backslash I}(l+\delta) \leq 2 \sqrt{\delta / l}
$$

if $\delta \leq l / 4$. In our case, $g_{\mathbb{C} \backslash I_{q}}(z) \leq 2 \sqrt{2 b_{s} / b_{q+1}}=2 \sqrt{2} \exp \left(2^{q}-2^{s-1}\right)$. Since $q \leq s-2$, we have $g_{\mathbb{C} \backslash K}(z) \leq 2 \sqrt{2} \exp \left(-2^{s-2}\right)$, which does not exceed $(2-\sqrt{2})^{s}$ for $s \geq 4$. This gives (5.8) for the first case.

It remains to consider $z_{0} \in K \cap\left[0, b_{s-1}\right]$. Recall that in the main bound (5.3) we estimated Lagrange fundamental polynomials with interpolating points $\left(x_{k}\right)_{k=1}^{D_{m}}$. Let us compare distances from these points to $z$ and to the point $-b_{s-2}$. If $x_{j} \leq b_{s-1}$ then $\left|z-x_{j}\right| \leq\left|z-z_{0}\right|+\left|z_{0}-x_{j}\right| \leq b_{s}+b_{s-1}<$ $b_{s-2}<b_{s-2}+x_{j}$. Otherwise, $x_{j} \geq a_{s-2}$ and $\left|z-x_{j}\right| \leq\left|z-z_{0}\right|+x_{j}=b_{s}+x_{j}<$ $b_{s-2}+x_{j}$.

It follows that $\left|L_{k}(z)\right|=\prod_{j=1, j \neq k}^{D_{m}}\left|z-x_{j}\right| /\left|x_{k}-x_{j}\right|<\left|L_{k}\left(-b_{s-2}\right)\right|$. Here, $s-2 \geq 2$, so we can apply (5.3). Arguing as above, we can generalize (5.1) to $(5.8)$.

6. On Totik's bound. In 2006 V. Totik [T, Th. 2.2] obtained the following remarkable estimate of the Green function (we formulate it for a compact set $K \subset[0,1])$ :

$$
g_{\mathbb{C} \backslash K}(-\delta) \leq C \sqrt{\delta} \exp \left(D \int_{\delta}^{1} \frac{\Theta_{K}^{2}(t)}{t^{3}} d t\right) \log \frac{2}{\operatorname{cap}(K)} .
$$

Here, $C, D$ are absolute constants, it is supposed that $K$ is not polar, and the function $\Theta_{K}$ is defined in our case as $\Theta_{K}(t)=m([0, t] \backslash K)$, where $m$ stands for the linear Lebesgue measure. In the case $\mathcal{I}_{\Theta_{K}}:=\int_{0}^{1} \Theta_{K}^{2}(t) t^{-3} d t<\infty$, the Green function $g_{\mathbb{C} \backslash K}$ has Lip $\frac{1}{2}$ smoothness, which is optimal for compact sets on $\mathbb{R}$. V. Totik proved that the condition of convergence of the integral 
is sharp: given function $\Theta$ with $\mathcal{I}_{\Theta}=\infty$, there exists a set $K$ with $\Theta_{K} \leq \Theta$ whose Green function is not in $\operatorname{Lip} \frac{1}{2}$ at the origin.

Thus, the estimation above is very appropriate to analyze boundary behavior of the Green functions with optimal smoothness. However, for compact sets with divergent $\mathcal{I}_{\Theta_{K}}$ the general estimate may be rough, because of uncontrollable constant $D$. For example in our case, $\int_{b_{s}}^{b_{1}} \Theta_{K}^{2}(t) t^{-3} d t>2^{s}-2$, so the right side of (6.1) exceeds $C b_{s}^{-D+1 / 2}$ for $\delta=b_{s}$.

Neither can the previous general bound of the Green functions by M. Tsuji [Ts, Th. III.67] be applied for the compact set considered in the paper. In fact, (6.1) is a refinement of the estimate by M. Tsuji.

It is also interesting to apply the lower bound by V. Andrievskii ([A1] or Th. 2.3 in [A2]) to our case. We get $g_{\mathbb{C} \backslash K}\left(-b_{s}\right)>\frac{1}{16} b_{s}^{1 / 2-\varepsilon}$ with rather small $\varepsilon$.

7. Markov's factors. Let us show that for $\gamma_{1}=\log 2 / \log (2+\sqrt{2})$ and some constant $C$ we have

$$
\exp n^{\gamma_{1}} \leq M_{n}(K) \leq \exp \left(C n^{\gamma_{1}}\right) \quad \text { for } n \in \mathbb{N} .
$$

Suppose that for some increasing continuous function $F$ we have the bound $g_{\mathbb{C} \backslash K}(z) \leq F(\delta)$ for $\operatorname{dist}(z, K) \leq \delta$. The application of the Cauchy formula for $P^{\prime}$ and the Bernstein-Walsh inequality gives (see e.g. [AG]) the estimate

$$
M_{n}(K) \leq \inf _{\delta} \delta^{-1} \exp [n F(\delta)] .
$$

In our case $F(\delta)=C \varphi^{\gamma}(\delta)$ and the value $\delta$ with $\left(\log \frac{1}{\delta}\right)^{1+\gamma}=C n$ gives the desired upper bound of $M_{n}(K)$.

On the other hand, fix $n \in \mathbb{N}$ and $m$ with $N_{m} \leq n<N_{m+1}$, where $N_{m}$ is given in Lemma 4.1(5). For the polynomial $P=P_{N_{m}}$ from Section 2 we have $\left|P^{\prime}(0)\right|=b_{m}^{-1}=\exp 2^{m}$ and $|P|_{K} \leq 1$, by Lemma 4.2.

Since the sequence $\left(M_{n}(K)\right)$ is nondecreasing, we get $M_{n}(K) \geq M_{N_{m}}(K)$ $\geq\left|P^{\prime}(0)\right| /|P|_{K} \geq \exp 2^{m}$. The last value exceeds $\exp N_{m+1}^{\gamma_{1}}$, since $N_{m+1}<$ $(2+\sqrt{2})^{m}$. This completes the proof of Corollary 2.2 as $N_{m+1}>n$.

\section{References}

[AG] M. Altun and A. Goncharov, On smoothness of the Green function for the complement of a rarefied Cantor-type set, Constr. Approx. 33 (2011), 265-271.

[A1] V. V. Andrievskii, On the Green function for a complement of a finite number of real intervals, Constr. Approx. 20 (2004), 565-583.

[A2] V. V. Andrievskii, Constructive function theory on sets of the complex plane through potential theory and geometric function theory, Surveys Approx. Theory 2 (2006), 1-52. 
[CG] T. Carroll and S. Gardiner, Lipschitz continuity of the Green function in Denjoy domains, Ark. Mat. 46 (2008), 271-283.

[Gol] G. M. Goluzin, Geometric Theory of Functions of a Complex Variable, Amer. Math. Soc., Providence, RI, 1969.

[G1] A. Goncharov, A compact set without Markov's property but with an extension operator for $C^{\infty}$ functions, Studia Math. 119 (1996), 27-35.

[G2] A. P. Goncharov, On the explicit form of an extension operator for $C^{\infty}$-functions, East J. Approx. 2 (2001), 179-193.

[N] R. Nevanlinna, Analytic Functions, Springer, 1970.

[P] C. Pommerenke, Univalent Functions, Vandenhoeck and Ruprecht, 1975.

[RR] T. Ransford and J. Rostand, Hölder exponents of Green's functions of Cantor sets, Comput. Methods Funct. Theory 1 (2008), 151-158.

[R] T. J. Rivlin, The Chebyshev Polynomials, 2nd ed., Wiley, New York, 1990.

[ST] H. Stahl and V. Totik, General Orthogonal Polynomials, Cambridge Univ. Press, 1992.

[T] V. Totik, Metric properties of harmonic measures, Mem. Amer. Math. Soc. 184 (2006), no. 867.

[Ts] M. Tsuji, Potential Theory in Modern Function Theory, Maruzen, Tokyo, 1959.

[W] N. Wiener, The Dirichlet problem, J. Math. Phys. 3 (1924), 127-146.

Serkan Celik

TÜBITAK, UEKAE P.K. 74

41470 Gebze, Kocaeli, Turkey

E-mail: serkanc@uekae.tubitak.gov.tr
Alexander Goncharov Department of Mathematics Bilkent University 06800 Ankara, Turkey E-mail: goncha@fen.bilkent.edu.tr

Received 30.7.2011

and in final form 17.1.2012 and 22.5.2012 